\title{
Bioluminescent Mucor circinelloides - a promising new tool to study mucormycosis and antifungal drug efficacy
}

Ulrike Binder'1, María Isabel Navarro-Mendoza², Francisco E. Nicolás², Cornelia Lass-Flörl1' and Victoriano Garre²

${ }^{1}$ Division of Hygiene and Medical Microbiology, Mecial University Innsbruck, Austria, ${ }^{2}$ Department of Genetics and Microbiology, Faculty of Biology, University of Murcia, Murcia, Spain

\section{Background}

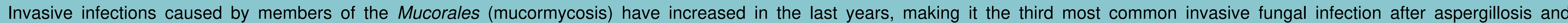
candidiasis. Despite this increasing clinical relevance, little is known about the establishment of disease, its progression and successful therapy.

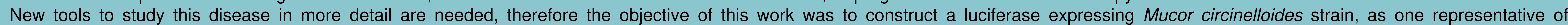

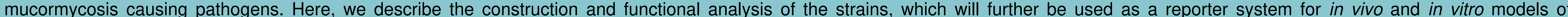
Mucorales infections.

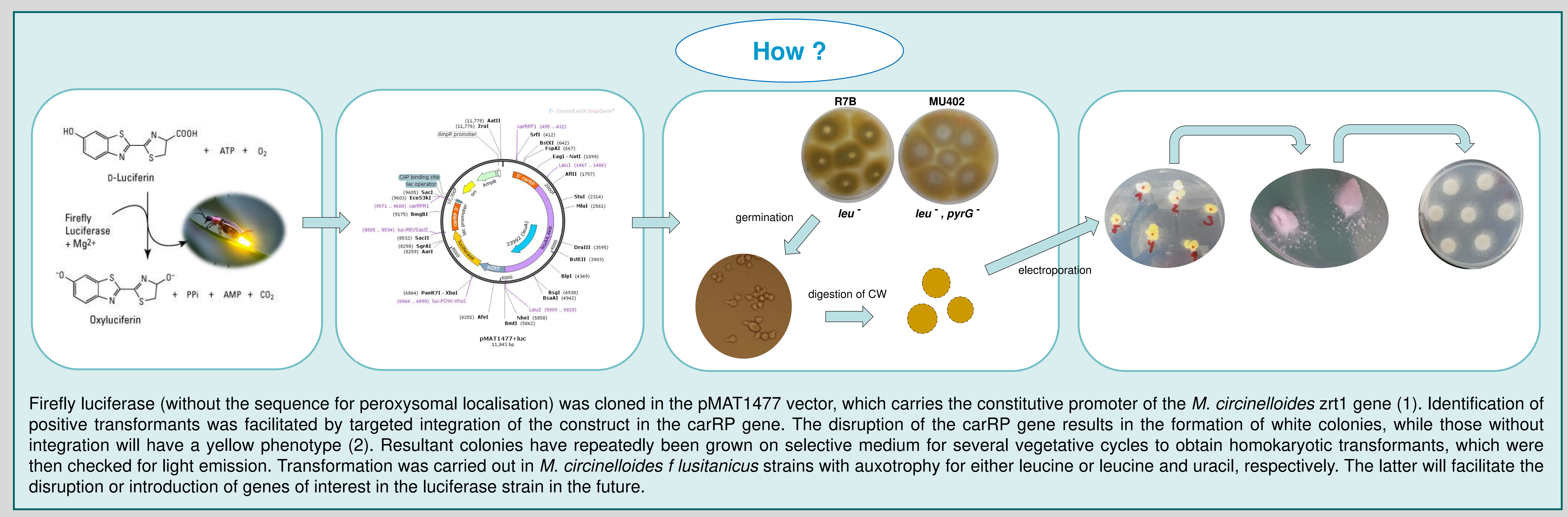

Functional analysis of luciferase containing M. circinelloides strains

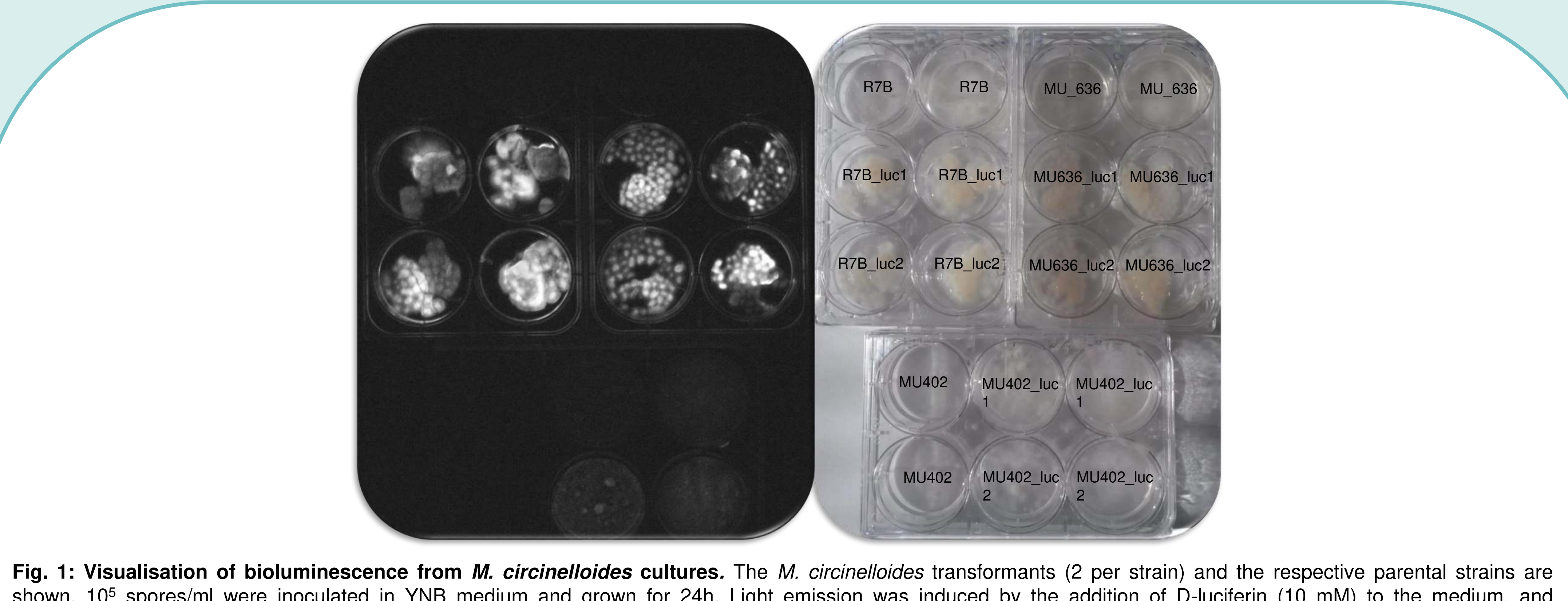

Fig. 1: Visualisation of bioluminescence from $M$. circinelloides cultures. The $M$. circinelloides transformants $(2$ per strain) and the respective parental strains are
shown. $10^{5}$ sisation shown. $10^{5}$ spores/ml were inoculated in YNB medium and grown for 244 . Light emission was induced by the addition of D-luciferin (10 mM
bioluminescence images of the cultures were acquired by a monochrome scientific grade CCD camera (BIO-VISION 3000 imaging system, left panel).

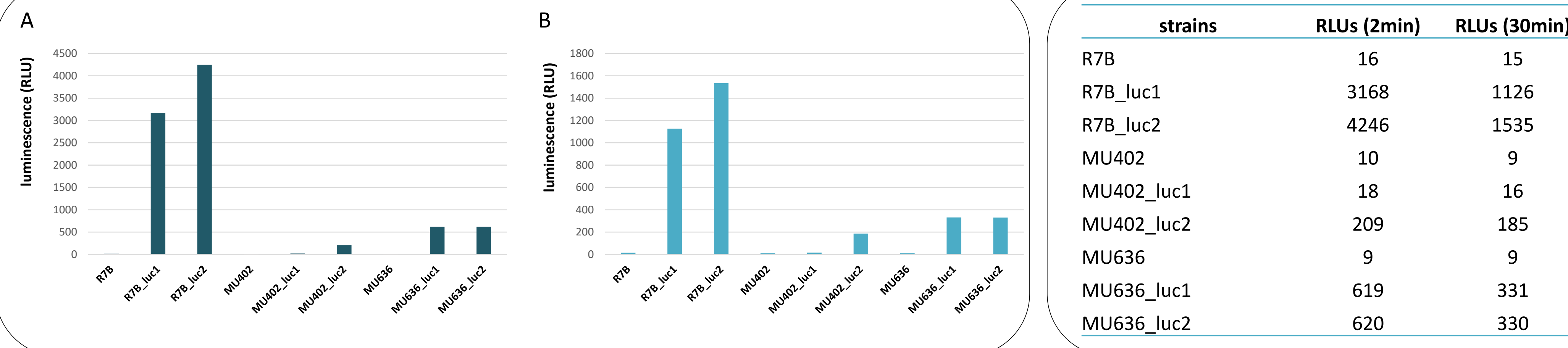

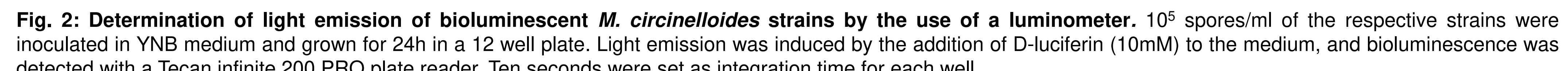

firefly luciferase is expressed in M. circinelloides and light emission can be detected

signal intensity differs depending on the recipient strain and correlates to fungal biomass

signal intensity is moderately stable over 30 min
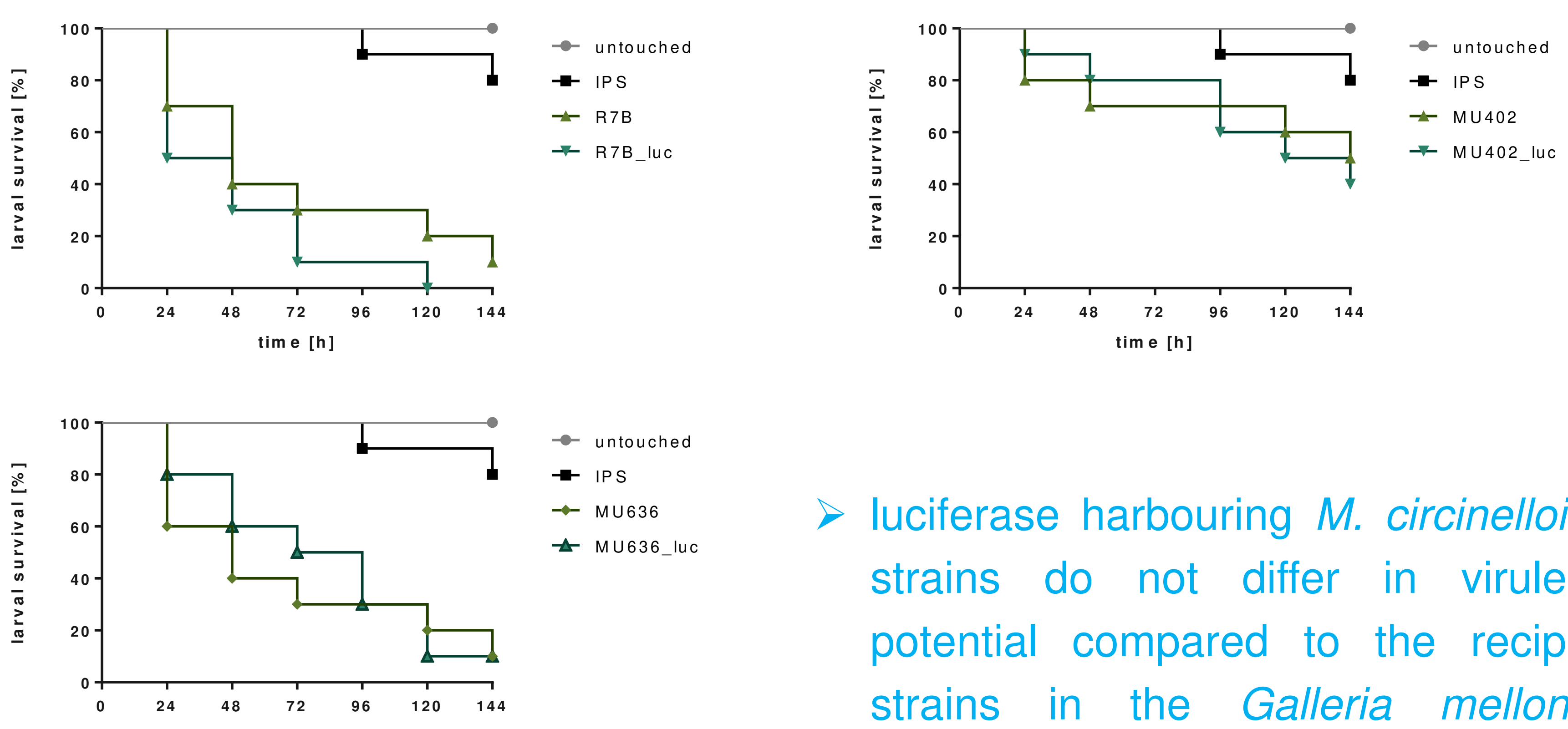

Fig. 3: Survival of larvae infected with $M$. circinelloides strains. incubated at $30^{\circ}$ C. Survival was monitored every 24 h up to $144 h$ h. Untouched larvae and larvae injected with IPS buffer served as controls. Results are express
experiments (60 larvae in total). Table 2: Susceptibility of $M$. circinelloides strains to azoles
and amphotericin B. Susceptibility testing was carried out
according to EUCAST guidlines in RPMI 1640 medium.

\begin{tabular}{lcccc} 
& \multicolumn{4}{c}{ MIC (Mg/ml) } \\
Strains & POSA & ITRA & ISA & AMB \\
\hline R7B & $>16$ & $>8$ & $>4$ & 2 \\
R7B_luc1 & $>16$ & $>8$ & $>4$ & 1 \\
R7B_luc2 & $>16$ & $>8$ & $>4$ & 2 \\
MU636 & $>16$ & $>8$ & $>4$ & 1 \\
MU636_luc1 & $>16$ & $>8$ & $>4$ & 2 \\
MU636_luc2 & $>16$ & $>8$ & $>4$ & 2 \\
MU402 & $>16$ & $>8$ & $>4$ & 2 \\
MU402_luc1 & $>16$ & $>8$ & $>4$ & 2 \\
MU402_luc2 & $>16$ & $>8$ & $>4$ & 2 \\
\hline
\end{tabular}

luciferase harbouring $M$. circinelloides strains do not differ in virulence potential compared to the recipient strains in the Galleria mellonella infection mode

\section{Conclusion and Outlook}

- firefly luciferase is expressed in M. circinelloides but signal intensity is rather low compared to other molds, therefore :

$>$ increase level of light expression by generating strains containing the firefly luciferase gene, codon optimized for expression in Mucor circinelloides

$\rightarrow$ with the generation of bioluminescent Mucorales strains we plan to

$>$ exploit novel methods of in vitro susceptibility testing

$>$ adapt alternative in vivo models (Galleria mellonella) and murine models to check in detail the onset and progression of mucormycosis

$>$ generate bioluminescent deletion strains to determine genes relevant for virulence

$>$ adapt alternative in vivo models (Galleria mellonella) and murine models to screen for antifungal treatment efficacy/test novel compounds

$\rightarrow$ the in vivo visualization of the temporal and spatial progression of Mucorales infections by a non-invasive method, will provide new and valuable information

$\rightarrow$ and will reduce the number of experimental animals

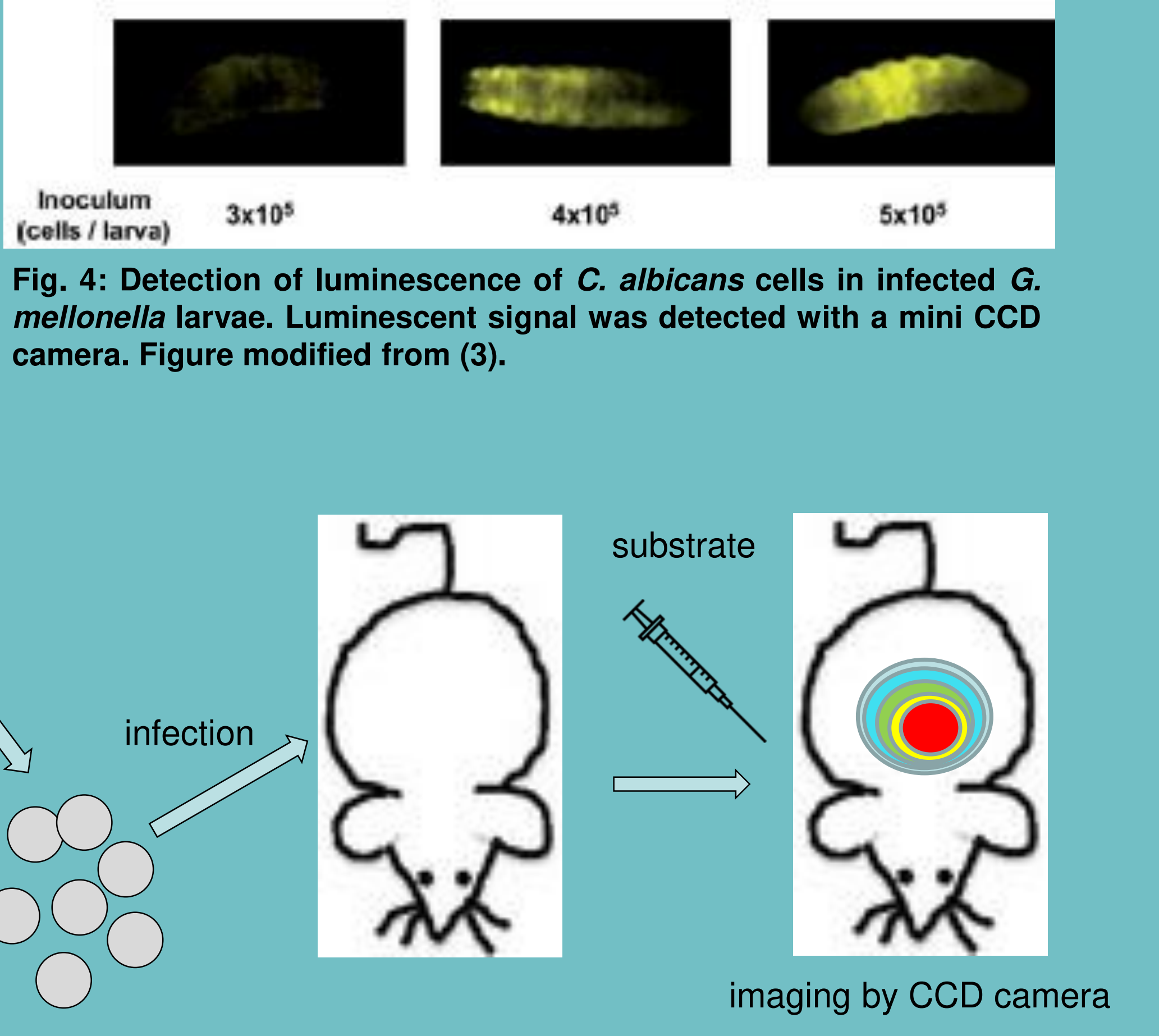

